\title{
Effective theory for QCD at finite temperature and density from strong coupling expansion
}

\author{
Michael Fromm, Jens Langelage, Stefano Lottini, Owe Philipsen* \\ Institut für Theoretische Physik, Johann Wolfgang Goethe-Universität Frankfurt, \\ 60438 Frankfurt am Main, Germany \\ E-mail: \\ fromm, langelage, lottini, philipseneth.physik.uni-frankfurt.de
}

\begin{abstract}
QCD at finite temperature and denisty remains intractable by Monte Carlo simulations for quark chemical potentials $\mu \gtrsim T$. It has been a long standing problem to derive effective theories from QCD which describe the phase structure of the former with controlled errors. We propose a solution to this problem by a combination of analytical and numerical methods. Starting from lattice QCD with in Wilson's formulation, we derive an effective action in terms of Polyakov loops by means of combined strong coupling and hopping expansions. The theory correctly reflects the centre-symmetry in the pure gauge limit and its breaking through quarks. It is valid for heavy quarks and lattices up to $N_{\tau} \sim 6$. Its sign problem can be solved and we are able to calculate the deconfinement transition of QCD with heavy quarks for all chemical potentials.
\end{abstract}

International Workshop on QCD Green's Functions, Confinement and Phenomenology, September 05-09, 2011

Trento Italy

*Speaker. 


\section{Introduction}

QCD at finite temperature and baryon density remains a challenge on the lattice since direct Monte Carlo simulations are prohibited by the sign problem, i.e. a complex fermion determinant, for non-vanishing quark chemical potential $\mu$. Existing workarounds based on reweighting, Taylor expansions in $\mu / T$ or simulations at imaginary chemical potential followed by analytic continuation all introduce additional systematic errors and require $\mu / T \lesssim 1$ in order to be valid. For an elementary introduction, see [1]. As a consequence, the QCD phase diagram remains largely unknown.

In this situation it is attractive or even mandatory to think about effective theories which should simplify computational problems while maintaining control over systematic errors. An example is dimensional reduction at finite temperature [2]. The approach relies on a sufficient scale separation $g T \ll \pi T$ between hard and soft modes in the thermal theory. The former then get integrated out perturbatively to produce a $3 \mathrm{~d}$ effective theory for the soft modes, which can be readily simulated on the lattice. Effective theories of the electroweak sector of the Standard Model made the correct predictions for the electroweak phase diagram [3], which were confirmed by $4 \mathrm{~d}$ lattice simulations [4]. The same approach applied to Yang-Mills theory is unable to describe the deconfinement transition, since the perturbative step explicitly breaks the centre symmetry of Yang-Mills theory [5]. Alternatively, there are efforts to write down the most general centre-symmetric $3 \mathrm{~d}$ effective action for the soft modes [6], and fix the couplings my perturbative or even non-perturbative matching calculations [7]. However, for $S U(3)$ this remains a difficult task due to the number of matching coefficients.

In this contribution we summarise a recent solution to this problem by means of strong coupling expansions on the lattice. Here we describe how to derive a centre-symmetric $3 \mathrm{~d}$ effective theory for thermal Yang-Mills theory and simulate it on the lattice. After successful completion of this step we include heavy quarks and compute the deconfinement transition as a function of quark mass and chemical potential.

\section{Yang-Mills theory}

\subsection{General strategy}

Consider the partition function of a $(3+1)$-dimensional lattice gauge field theory at finite temperature $\left(T=\frac{1}{a N_{\tau}}\right)$ with gauge group $S U(N)$ and Wilson's gauge action

$$
Z=\int\left[d U_{0}\right]\left[d U_{i}\right] \exp \left[\frac{\beta}{2 N} \sum_{p}\left(\operatorname{tr} U_{p}+\operatorname{tr} U_{p}^{\dagger}\right)\right], \quad \beta=\frac{2 N}{g^{2}} .
$$

Finite temperature and the bosonic nature of the degrees of freedom imply the use of periodic boundary conditions in the time direction. Finer lattices correspond to larger $N_{\tau}$ for fixed physics. We now integrate out the spatial degrees of freedom and get schematically [8]

$$
\begin{aligned}
Z & =\int\left[d U_{0}\right] \exp \left[-S_{\text {eff }}\right] \\
-S_{\text {eff }} & =\ln \int\left[d U_{i}\right] \exp \left[\frac{\beta}{2 N} \sum_{p}\left(\operatorname{tr} U_{p}+\operatorname{tr} U_{p}^{\dagger}\right)\right] \equiv \lambda_{1} S_{1}+\lambda_{2} S_{2}+\ldots .
\end{aligned}
$$




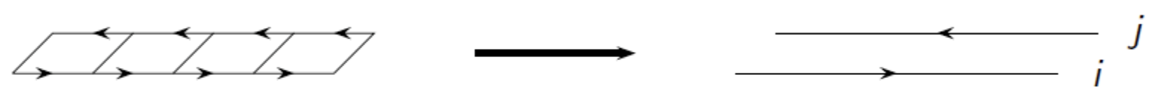

Figure 1: First graph with a nontrivial contribution after spatial integration for a lattice with temporal extent $N_{\tau}=4$. Four plaquettes in the fundamental representation lead to an interaction term involving two adjacent fundamental Polyakov loops $L_{i}$ and $L_{j}$.

We expand around $\beta=0$ and arrange the effective couplings $\lambda_{n}=\lambda_{n}\left(\beta, N_{\tau}\right)$ in increasing order in $\beta$ of their leading terms. Thus, the $\lambda_{n}$ become less important the higher $n$. As we shall see, the interaction terms $S_{n}$ depend only on Polyakov loops

$$
L_{j} \equiv \operatorname{tr} W_{j} \equiv \operatorname{tr} \prod_{\tau=1}^{N_{\tau}} U_{0}\left(\vec{x}_{j}, \tau\right) .
$$

With sufficiently accurate knowledge of the relations $\lambda_{n}\left(\beta, N_{\tau}\right)$, we are able to convert the couplings of the three-dimensional theory to those of the full theory. Determining the critical parameters $\lambda_{n, c}$ of the effective theory then gives a whole array of critical $\beta_{c}\left(N_{\tau}\right)$ for - in principle - all $N_{\tau}$. In the following we calculate strong coupling, i.e. small $\beta$, expansions of the leading $\lambda_{n}$.

Since the calculations are quite similar for different numbers of colours, we now specialise our derivation to the physically interesting case of $S U(3)$. For more details and the simpler case of $S U(2)$, see [9]. Using the character expansion as described e.g. in [10,11], the effective action according to Eq. (2.2) can be written as

$$
-S_{\mathrm{eff}}=\ln \int\left[d U_{i}\right] \prod_{p}\left[1+\sum_{r \neq 0} d_{r} a_{r}(\beta) \chi_{r}\left(U_{p}\right)\right],
$$

where the sum extends over all irreducible representations $r$ with dimension $d_{r}$ and character $\chi_{r}$. The expansion coefficients $a_{r}(\beta)$ are known as series for $S U(3)$ or in closed form for $S U(2)$ [10] and in the following we use $u \equiv a_{f}$ as expansion parameter instead of $\beta$ for its better apparent convergence. Our task is then to group together all graphs yielding the same interaction terms up to some order in $\beta$.

The leading order result of the effective action has first been calculated in [12] and corresponds to a sequence of $N_{\tau}$ plaquettes that wind around the lattice in temporal direction, cf. Fig. 1.. Its contribution is given by:

$$
\lambda_{1} S_{1}=u^{N_{\tau}} \sum_{<i j>} L_{i} L_{j}
$$

Hence, to leading order the first coupling of the effective theory is $\lambda_{1}\left(u, N_{\tau}\right)=u^{N_{\tau}}$. Corrections consist of geometrical decorations containing additional plaquettes, e.g. a cube sitting on top of the previous graph, etc. Calculating seven non-trivial orders, we find for the leading coupling

$$
\begin{aligned}
\lambda_{1}\left(N_{\tau} \geq 6, u\right)=u^{N_{\tau}} \exp \left[N _ { \tau } \left(4 u^{4}\right.\right. & +12 u^{5}-14 u^{6}-36 u^{7} \\
& \left.\left.+\frac{295}{2} u^{8}+\frac{1851}{10} u^{9}+\frac{1055797}{5120} u^{10}\right)\right] .
\end{aligned}
$$



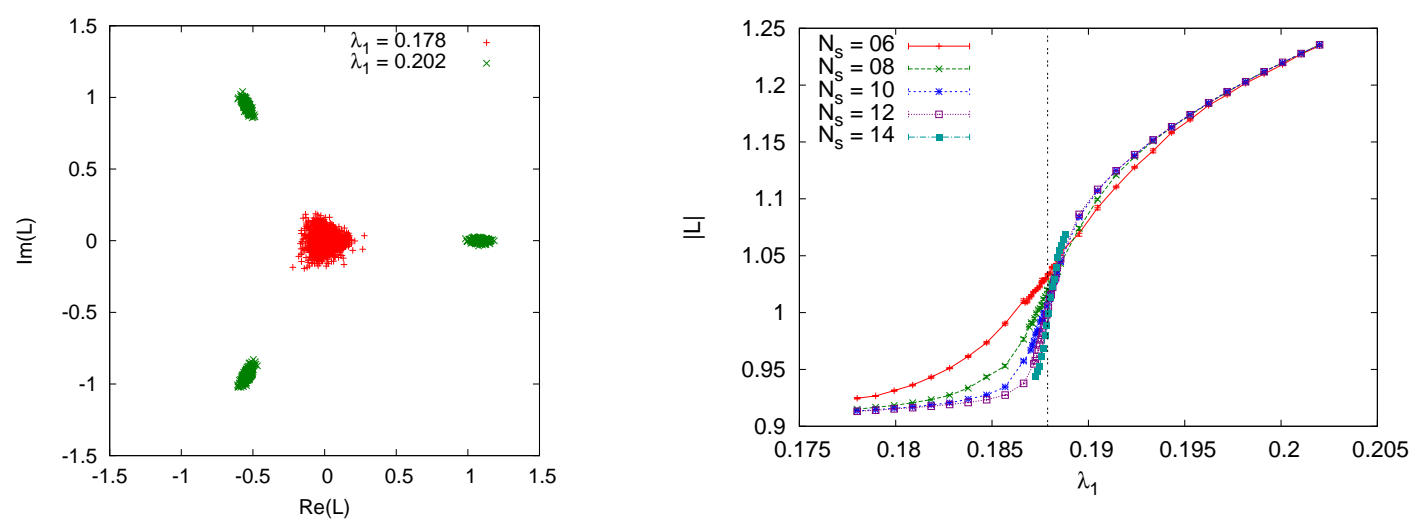

Figure 2: Left: Distribution of $L$ for small and large $\lambda_{1}$ on a lattice with $N_{s}=6$. Right: Expectation value of $|L|$. The vertical line marks the infinite-volume transition.

For smaller $N_{\tau}$ some lower coefficients change since some graphs do not fit into the lattice, detailed expressions for those cases are given in [9]. Inspection of higher order terms shows that one can arrange a subclass as

$$
\sum_{<i j>}\left(2 \lambda_{1} \operatorname{Re}\left(L_{i} L_{j}^{*}\right)-\frac{4 \lambda_{1}^{2}}{2} \operatorname{Re}\left(L_{i} L_{j}^{*}\right)^{2}+\ldots\right)=\sum_{<i j>} \ln \left(1+2 \lambda_{1} \operatorname{Re}\left(L_{i} L_{j}^{*}\right)\right) .
$$

In a similar fashion we have calculated the leading terms of some additional couplings. For the first terms of the next-to-nearest neighbour coupling $\lambda_{2}\left(N_{\tau}, u\right)$ we find

$$
\lambda_{2}\left(N_{\tau} \geq 8, u\right)=u^{2 N_{\tau}}\left[N_{\tau}\left(N_{\tau}-1\right) u^{2}\right],
$$

while the leading coupling of adjoint loops is (valid for $N_{\tau} \geq 2$ )

$$
\lambda_{a}=v^{N_{\tau}}\left(1+N_{\tau} \frac{3}{2} \frac{u^{6}}{v}+\ldots\right), \quad v=\frac{9}{8} u^{2}-\frac{9}{8} u^{3}+\frac{81}{32} u^{4}+\ldots
$$

A further simplification is achieved by using the trace of the Polyakov loops for the path integral measure as degrees of freedom (complex numbers instead of matrices), and rewrite the one-coupling partition function as

$$
Z_{\mathrm{eff}}=\int\left(\prod_{i} d L_{i} e^{V_{i}}\right) \prod_{<i j>}\left(1+2 \lambda_{1} \operatorname{Re} L_{i} L_{j}^{*}\right), \quad V_{i}=\frac{1}{2} \ln \left(27-18\left|L_{i}\right|^{2}+8 \operatorname{Re}\left(L_{i}^{3}\right)-\left|L_{i}\right|^{4}\right) .
$$

We thus have arrived at a simple, centre-symmetric theory of complex scalars in three dimensions.

\subsection{Numerical analysis of effective theories for hot Yang-Mills}

The effective theory allows for straightforward Monte Carlo simulations using a standard Metropolis update. Our first task is to establish the phase structure of the effective theory, where we focus on the physically interesting case of $S U(3)$. Based on the global $Z(3)$ symmetry of the model, one expects spontaneous breaking of that symmetry for some critical value of the coupling $\lambda_{1, c}$. Fig. 2 shows the behaviour of the field variable $L$ as a function of $\lambda_{1}$. As expected from the $4 \mathrm{~d}$ parent theory, there is indeed a transition from a disordered or mixed phase, with values of $L$ scattering about zero, to an ordered phase at large coupling where the three $Z(3)$-phases are populated 

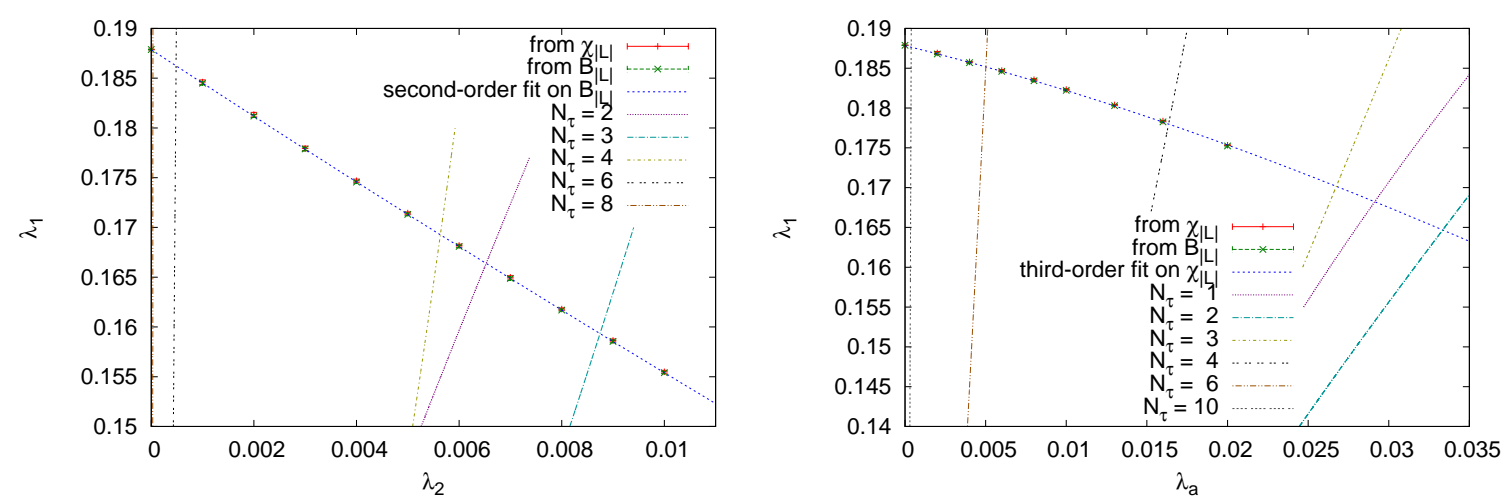

Figure 3: Critical line in the two-coupling space, determined from $\chi(|L|)$. Dashed lines give the parameter space representing a $4 \mathrm{~d}$ theory with fixed $N_{\tau}$.

separately. In the thermodynamic limit, one of these vacua will be chosen and the symmetry is broken spontaneously. Correspondingly, the expectation value of $|L|$ rises abruptly at some critical coupling $\lambda_{1 c}$, as shown in Fig. 2 (right). On a finite size lattice, the phase transition is smoothed out, non-analyticities are approached gradually with growing volume, as the figure illustrates.

In order to locate the critical coupling, we looked amongst others at peaks of the susceptibility

$$
\chi(|L|)=N_{s}^{3}\left\langle(|L|-\langle|L|\rangle)^{2}\right\rangle
$$

with $|L|=\left|\sum_{i} L_{i}\right|$ After identifying a pseudo-critical $\lambda_{1, c}\left(N_{s}\right)$ for a number of finite systems, the relation $\lambda_{1 c}\left(N_{s}\right)=\lambda_{1 c}+b N_{s}^{-1 / v}$ is used to extrapolate to the thermodynamic limit. The data are compatible with $v=1 / 3$ for the $S U$ (3) first-order transitions and, in the $S U(2)$ case, the $3 \mathrm{~d}$ Ising value $v=0.63002$. Note that due to having only a $3 \mathrm{~d}$ scalar theory, even an intricate finite size scaling analysis can be done in just a few days on a desktop PC.

\begin{tabular}{|c||c|c|c||c|}
\hline$N_{\tau}$ & $\lambda_{1}$ & $\left(\lambda_{1}, \lambda_{2}\right)$ & $\left(\lambda_{1}, \lambda_{a}\right)$ & $4 \mathrm{~d} \mathrm{YM}$ \\
\hline 1 & $2.7828(4)$ & - & $2.529(6)$ & $2.703(4)$ \\
2 & $5.1839(2)$ & $5.0174(4)$ & $5.003(5)$ & $5.10(5)$ \\
3 & $5.8488(1)$ & $5.7333(3)$ & $5.780(2)$ & $5.55(1)$ \\
4 & $6.09871(7)$ & $6.0523(1)$ & $6.0748(6)$ & $5.6925(2)$ \\
6 & $6.32625(4)$ & $6.32399(3)$ & $6.3225(1)$ & $5.8941(5)$ \\
8 & $6.43045(3)$ & $6.43033(2)$ & $6.42971(7)$ & $6.001(25)$ \\
10 & $6.49010(2)$ & $6.49008(2)$ & $6.48991(6)$ & $6.160(7)$ \\
12 & $6.52875(2)$ & $6.52874(1)$ & $6.52869(5)$ & $6.268(12)$ \\
14 & $6.55584(2)$ & $6.55583(1)$ & $6.55580(4)$ & $6.383(10)$ \\
16 & $6.57588(1)$ & $6.57587(1)$ & $6.57585(3)$ & $6.45(5)$ \\
\hline
\end{tabular}

Table 1: Critical couplings $\beta_{c}$ for $S U(3)$ from different effective theories compared to simulations of the $4 \mathrm{~d}$ theory $[13,14]$. The $N_{\tau}=1$ Monte Carlo value is from our own simulation with standard Cabibbo-Marinari plaquette update. 

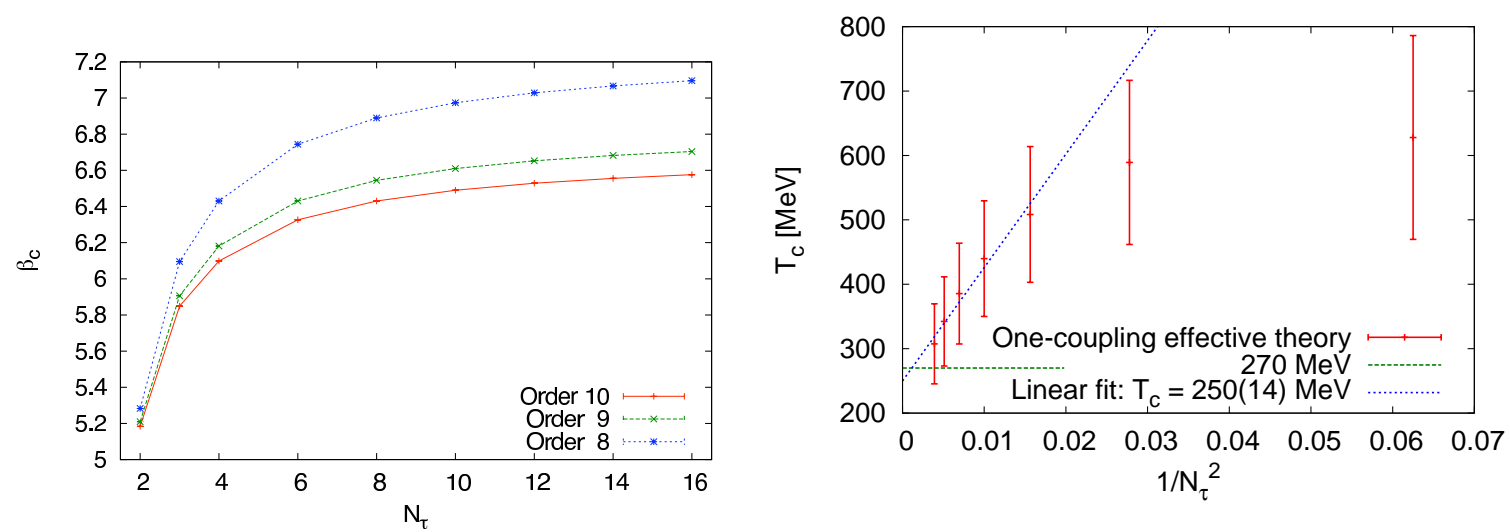

Figure 4: Left: Critical coupling for the $S U(3)$ pure gauge transition, calculated from $\lambda_{1 c}$ using the maps Eq. 2.6. Right: Critical temperature for the pure gauge deconfinement transition, extracted from the $3 \mathrm{~d}$ effective theory, Eq. (2.10).

Next we investigated the influence of a second coupling on the effective theory, i.e. the nextto-nearest-neighbour coupling $\lambda_{2}$ and the adjoint loop coupling $\lambda_{a}$. In these two-dimensional parameter spaces, there is a critical line separating the symmetric and the broken phases. However, for a given $N_{\tau}$, only a one-dimensional manifold in this space represents the image of the original gauge theory, since both couplings are functions of $u$ only. The results for both cases are shown in Fig. 3. By plotting the family of curves corresponding to a given $N_{\tau}$, one sees that the latter accumulate towards vanishing second coupling as $N_{\tau}$ increases, i.e. as the lattice gets finer. For continuum physics they may be safely neglected.

Having established the critical couplings for our effective theories we are now ready to map them back to the original thermal Yang-Mills theories by inverting the couplings $\lambda_{i c}\left(\beta, N_{\tau}\right) \rightarrow$ $\beta_{c}\left(\lambda_{i}, N_{\tau}\right)$. In Table 1 we collect the values for the critical gauge couplings obtained in this way and compare them to the values obtained from simulations of the full $4 \mathrm{~d}$ theory. The agreement is remarkable in all cases and even better in the case of $S U(2)$. Interestingly, there appears to be a 'region of best agreement', with the deviation growing both for small and large $N_{\tau}$. We ascribe this to the fact that there are two competing systematic errors, as discussed earlier: the validity of the strong coupling series for a given coupling $\lambda_{i}$ is better the smaller $\beta$ and hence $N_{\tau}$, whereas the truncation of the next-to-nearest neighbour interactions gains validity with growing $N_{\tau}$. In particular in the case of $S U(3)$, there appears to be a cancellation of the two kinds of systematics, rendering the effective description better for the original theory on finer lattices.

The convergence properties of the strong coupling expansion in our "observable" $\beta_{c}$ are shown in Fig. 4 (left) for the three highest orders calculated in $S U$ (3). Even on fine lattices we observe reasonable convergence, which encourages us to attempt a continuum extrapolation. Supplying a scale by using the non-perturbative beta function $a(\beta)$ from [15], we convert the critical couplings to temperatures and plot them in Fig. 4 (right). The error bars give the difference between the two highest orders of the strong coupling expansion. The onset of the $N_{\tau}^{-2} \sim a^{2}$ scaling region is clearly visible providing a continuum extrapolation within $\sim 10 \%$ of the known result. Note that all data points stem from a single $3 \mathrm{~d}$ simulation. 


\section{QCD with heavy fermions}

\subsection{The hopping parameter expansion}

Let us now summarise the inclusion of heavy fermions, details can be found in [16]. Heavy fermions are conveniently introduced using the hopping parameter expansion, which is described in $[10]$ at zero temperature and discussed in $[17,18]$ for finite temperature. The quark part of the action for $N_{f}$ mass-degenerate flavours with masses $M_{f}=M$ can then be written

$$
-S_{q}=-N_{f} \sum_{l=1}^{\infty} \frac{\kappa^{l}}{l} \operatorname{Tr} H[U]^{l}, \quad \kappa=\frac{1}{2 a M+8}, \quad H[U]_{y, x} \equiv \sum_{ \pm v} \delta_{y, x+\hat{v}}\left(1+\gamma_{v}\right) U_{v}(x),
$$

with $\gamma_{-v}=-\gamma_{v}$. Thus each hop to a neighbouring lattice site gives a power of the hopping parameter $\kappa$. The quark chemical potential $\mu$ is introduced as usual by a factor $\mathrm{e}^{a \mu}\left(\mathrm{e}^{-a \mu}\right)$ multiplying link variables in positive (negative) time direction [19]. The effective theory is obtained from the full theory in the same way as for Yang-Mills,

$$
\begin{aligned}
Z & =\int\left[d U_{0}\right]\left[d U_{i}\right] \exp \left[-S_{g}-S_{q}\right]=\int\left[d U_{0}\right] \exp \left[-S_{\text {eff }}\right], \\
-S_{\text {eff }} & =\ln \int\left[d U_{i}\right] \exp \left[-S_{g}-S_{q}\right] .
\end{aligned}
$$

We are now faced with a double series expansion in $u(\beta)$ and $\kappa$, i.e. the effective couplings depend on both parameters and $N_{\tau}$. Furthermore, quarks of finite mass lead to terms in the action which explicitly break the $Z(3)$ symmetry present in the pure gauge case. We may arrange this as

$$
-S_{\mathrm{eff}}=\sum_{i=1}^{\infty} \lambda_{i}\left(u, \kappa, N_{\tau}\right) S_{i}^{s}-2 N_{f} \sum_{i=1}^{\infty}\left[h_{i}\left(u, \kappa, \mu, N_{\tau}\right) S_{i}^{a}+\bar{h}_{i}\left(u, \kappa, \mu, N_{\tau}\right) S_{i}^{a, \dagger}\right] .
$$

The $\lambda_{i}$ are defined as the effective couplings of the $Z(3)$-symmetric terms $S_{i}^{s}$, whereas the $h_{i}$ multiply the asymmetric terms $S_{i}^{a}$. Consequently, only the latter are $\mu$-dependent and we recover pure gauge theory for $\kappa \rightarrow 0$, as in the full theory.

Similar to the case of pure gauge theory, graphs contributing to the cluster expansion have to wind around the lattice in the compact $\tau$-direction. Hence, the leading order contributions are Polyakov loops, and we can read off the leading-order couplings $h_{1}$ and $\bar{h}_{1}$ :

$$
h_{1} S_{1}^{a}+\bar{h}_{1} S_{1}^{a, \dagger}=-\sum_{\vec{x}}\left[\left(2 \kappa e^{a \mu}\right)^{N_{\tau}} L(\vec{x})+\left(2 \kappa e^{-a \mu}\right)^{N_{\tau}} L^{*}(\vec{x})\right] \rightarrow h_{1}=\left(2 \kappa e^{a \mu}\right)^{N_{\tau}} .
$$

Note the minus sign which is due to the anti-periodic boundary conditions for fermions. It is possible to sum up the contributions of all generalised Polyakov loops oriented in positive time direction,

$$
\exp \left\{-2 N_{f} \sum_{\vec{x}} \sum_{n=1}^{\infty}\left[\frac{(-1)^{n}}{n}\left(2 \kappa e^{a \mu}\right)^{n N_{\tau}} \operatorname{Tr}\left(W^{n}\right)\right]\right\}=\prod_{\vec{x}} \operatorname{det}\left[1+\left(2 \kappa e^{a \mu}\right)^{N_{\tau}} W\right]^{2 N_{f}},
$$

using $\exp \operatorname{Tr} \ln A \equiv \operatorname{det} A$, and similarly for the conjugate loop. The three-dimensional effective action to leading order in the hopping expansion corresponds to the static approximation and reads

$$
Z_{\mathrm{eff}}=\int\left[d U_{0}\right]\left(\prod_{<i j>}\left[1+2 \lambda_{1} \operatorname{Re} L_{i}^{*} L_{j}\right]\right)\left(\prod_{\vec{x}} \operatorname{det}\left[\left(1+h_{1} W_{\vec{x}}\right)\left(1+\bar{h}_{1} W_{\vec{x}}^{\dagger}\right)\right]^{2 N_{f}}\right) .
$$


Owe Philipsen
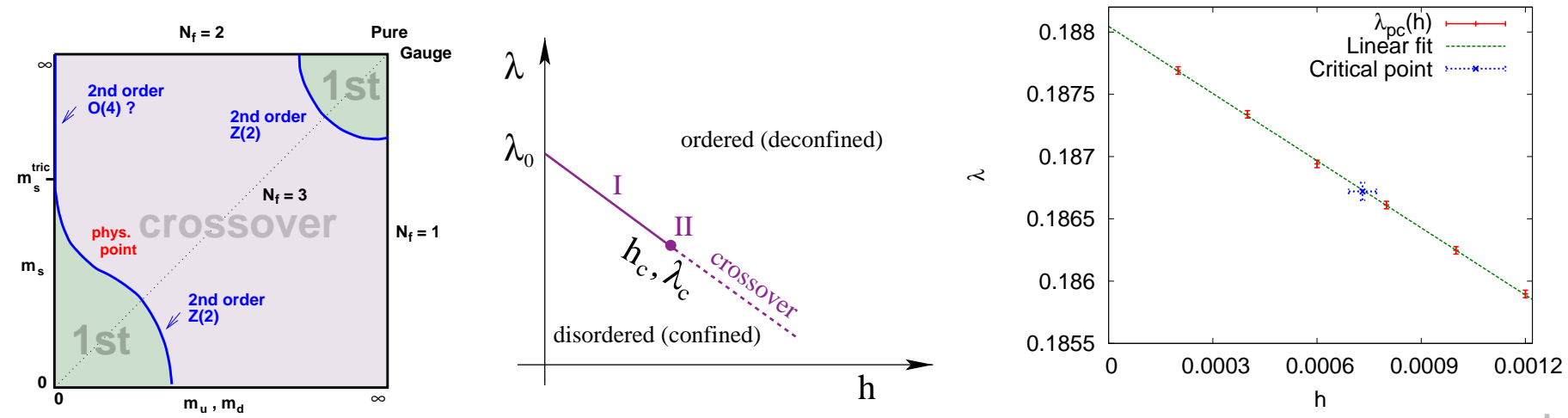

Figure 5: Schematic phase diagram for $\mu=0$. Left: Nature of the QCD thermal transition for different quark masses. Middle: Expected phase diagram for the effective theory corresponding to $\mu=0$. Right: Calculated phase diagram for $\mu=0$. Also the critical point is shown.

Using some identities for determinants and reverting to traced Polyakov loops again, this becomes

$$
\begin{aligned}
Q_{i}(h, \bar{h}) & =\left[\left(1+h L_{i}+h^{2} L_{i}^{*}+h^{3}\right)\left(1+\bar{h} L_{i}^{*}+\bar{h}^{2} L_{i}+\bar{h}^{3}\right)\right]^{2}, \\
Z_{\mathrm{eff}} & =\int\left(\prod_{i} d L_{i} e^{V_{i}} Q_{i}^{N_{f}}(h, \bar{h})\right) \prod_{<i j>}\left(1+2 \lambda \operatorname{Re} L_{i} L_{j}^{*}\right) .
\end{aligned}
$$

Going beyond the static approximation means to include also spatial hops, which get decorated with plaquettes. Including all corrections up to $\mathscr{O}\left(u^{n} \kappa^{m}\right)$, with $n+m=7$, we obtain

$$
\begin{aligned}
h_{1}\left(u, \kappa, N_{\tau} \geq 3\right)=\left(2 \kappa e^{a \mu}\right)^{N_{\tau}} \exp & {\left[6 N _ { \tau } \kappa ^ { 2 } u \left(\frac{1-u^{N_{\tau}-1}}{1-u}\right.\right.} \\
+ & \left.\left.4 u^{4}-8 \kappa^{2}+8 \kappa^{2} u+4 \kappa^{2} u^{2}-4 \kappa^{4}\right)\right] .
\end{aligned}
$$

Additional couplings turn out to be suppressed compared to this and we neglect them in the following. Since we thus keep only one coupling of each sort, we drop the index " 1 " for the remainder. Since the deconfinement transition at high temperature happens at small $h$, we can recover an arbitrary number of flavours by using the approximation $\operatorname{det}(1+h W)^{N_{f}} \approx \exp \left(N_{f} h L\right)$.

\subsection{The deconfinement transition at zero baryon density}

As a first application of the effective theory we investigate the deconfinement transition of QCD with heavy quarks as a function of quark mass and chemical potential. We begin by considering the case of zero baryon density, shown schematically in Fig. 5 (left). In the pure gauge limit, the deconfinement transition is of first order. Dynamical quarks at any fixed $N_{f}$ break the global $Z(3)$ symmetry of the QCD action explicitly. As a consequence, the phase transition weakens with decreasing quark masses until it vanishes at a critical point. For still lighter quarks the deconfinement transition is an analytic crossover. This behaviour is inherited by the effective theory. For a given $N_{f}$ and $\mu=0$, we have $h=\bar{h}$ and the effective theory has two couplings, $(\lambda, h)$. The first-order phase transition of the one-coupling theory extends to a first-order line with a weakening transition as $h$ increases. Eventually the transition vanishes at a critical point, as sketched in Fig. 5 (middle). 

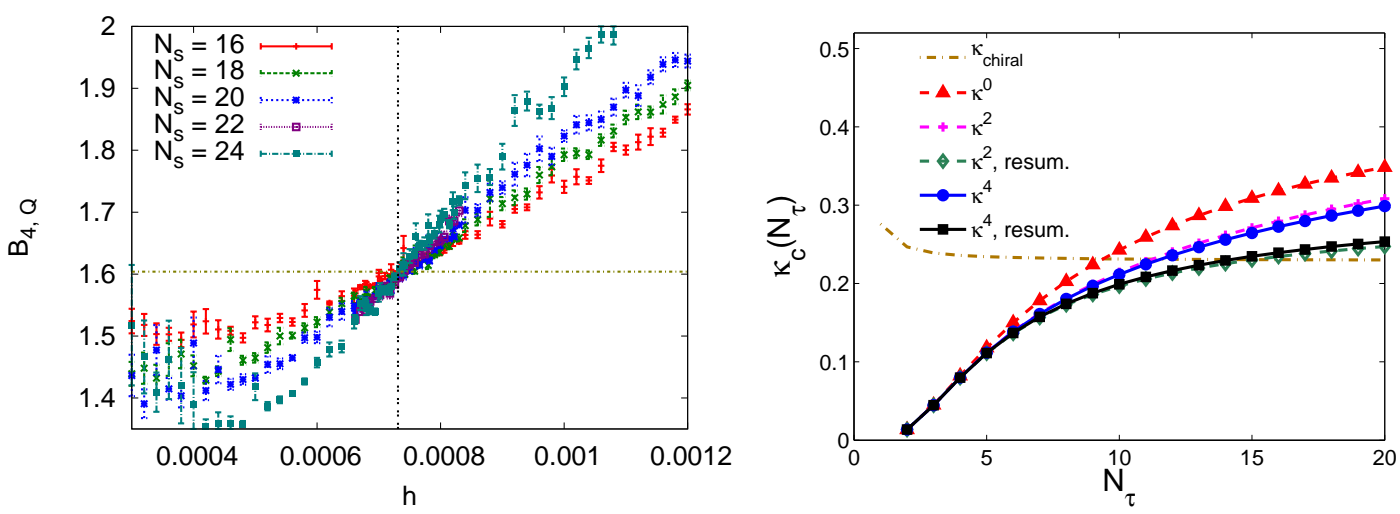

Figure 6: Left: Determination of the critical point as the crossing of the Binder cumulant on different volumes. Right: Critical hopping parameter $\kappa_{c}\left(N_{\tau}\right)$ for $N_{f}=1$.

In a slight sharpening of terminology, we now call the phase boundary pseudo-critical and only refer to the second order endpoint as "critical".

The numerical analysis proceeds in the same fashion as described in the pure gauge section with two couplings. The resulting phase boundary between the ordered and disordered phase is shown in Fig. 5 (right) and found to be linear in the small coupling $h$. In order to locate the critical endpoint we study the scaling of the fourth order Binder cumulant

$$
B_{4}(Q) \equiv\left\langle(Q-\langle Q\rangle)^{4}\right\rangle /\left\langle(Q-\langle Q\rangle)^{2}\right\rangle^{2},
$$

along the phase boundary in Fig. 6 (right). The crossing point provides us with the critical coupling $h_{c}$ and the value of the cumulant at the crossing point is consistent with the $3 \mathrm{~d}$ Ising value, as expected for a critical end point. We identify the critical point as

$$
\left(\lambda_{c}=0.18672(7), h_{c}=0.000731(40)\right) \text {. }
$$

Using the analytic expressions for the couplings, these values can be converted into those of the couplings $\beta_{c}, \kappa_{c}$. In order to compare with previous work, we approximate $M_{c} / T$ with the relation, valid for heavy fermions to leading order in the hopping expansion [20],

$$
\exp \left(-\frac{M}{T}\right) \simeq \frac{h}{N_{f}}
$$

The results for an $N_{\tau}=4$ lattice are collected in Table 2 and once more in good agreement with $4 \mathrm{~d}$ Monte Carlo simulations.

As in pure gauge theory, we may look at the continuum approach of $\kappa_{c}$, obtained by inverting Eq. (3.8), Fig. 6 (right). Also shown is the chiral critical hopping parameter, defined by the vanishing of the pion mass and evaluated for the critical gauge couplings, $\kappa_{c h}\left(u_{c}\left(N_{\tau}\right)\right)$. Since we are expanding around infinite quark masses, self-consistency requires $\kappa_{c} \ll \kappa_{c h}$. This suggests that our expansion is trustworthy to about $N_{\tau} \sim 6$.

\subsection{The deconfinement transition at finite baryon density}

For $\mu \neq 0$, we have $h \neq \bar{h}$ and need to consider the full parameter space of the effective theory, $(\lambda, h, \bar{h})$. The diagram in Fig. 5 (middle) turns three-dimensional, with a surface of first order phase 


\begin{tabular}{|c|c|c||c|c|}
\hline$N_{f}$ & $M_{c} / T$ & $\kappa_{c}\left(N_{\tau}=4\right)$ & $\kappa_{c}(4)$, Ref. [21] & $\kappa_{c}(4)$, Ref. [22] \\
\hline 1 & $7.22(5)$ & $0.0822(11)$ & $0.0783(4)$ & $\sim 0.08$ \\
2 & $7.91(5)$ & $0.0691(9)$ & $0.0658(3)$ & - \\
3 & $8.32(5)$ & $0.0625(9)$ & $0.0595(3)$ & - \\
\hline
\end{tabular}

Table 2: Location of the critical point for $\mu=0$ and $N_{\tau}=4$. The first two columns are our results the last two from the literature.
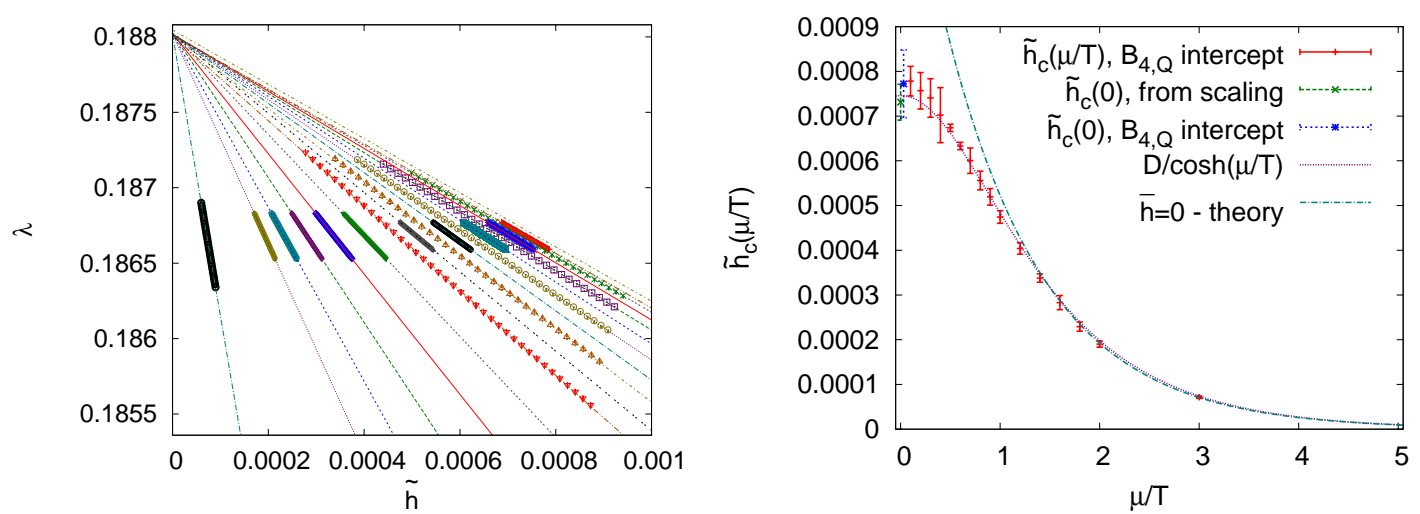

Figure 7: Left: Transition lines $\lambda_{p c}(\tilde{h}, \mu / T)$ for several values of $\mu / T$ between 0 (top) and 3.0 (bottom). Right: Critical line $\tilde{h}_{c}(\mu / T)$; the curves are fits to $\cosh ^{-1}(\mu / T)$ behaviour and the large- $\mu$ asymptotic limit.

transitions terminating in a critical line. Since we are interested in the change of the critical quark mass with chemical potential, it is convenient to introduce the parameter

$$
\tilde{h} \equiv h e^{-\mu / T}\left(=(2 \kappa)^{N_{\tau}} \text { to leading order in } \kappa\right)
$$

and to present our data in the parameter space $(\lambda, \tilde{h}, \mu / T)$.

For $\bar{h} \neq h$ the effective theory has a sign problem, just as the original theory. However, the sign problem is very mild and can be overcome by using the modulus of the fermion determinant in the path integral measure while reweighting in its phase. Moreover, the sign problem can be solved by going to a flux representation of the partition function, which can then be simulated by a worm algorithm. Details and a comparison of entirely agreeing results can be found in [16]. The numerical analysis then proceeds in complete analogy to the zero density case. The pseudo-critical lines $\lambda_{p} c(\tilde{h})$ for various choices of $\mu / T$ are shown in Fig. 7 (left). As in the zero density case, we observe perfectly linear behaviour due to the smallness of $\tilde{h}$. Again the critical point on each line is found by identifying where the Binder cumulant takes its critical value. The corresponding line of critical endpoints is shown in Fig. 7 (right).

Asymptotically large chemical potentials in the original lattice QCD are described by the limit

$$
\kappa \rightarrow 0, \mu \rightarrow \infty \text { with } \quad \kappa e^{\mu / T}=\text { const . }
$$

In the effective theory this corresponds to, $(\lambda, h, \bar{h}=0)$. The critical point in this case is easily found by the same techniques,

$$
\left.\left(\lambda_{c}, h_{c}\right)\right|_{\bar{h}=0}=(0.18668(2), 0.00142(2)) .
$$



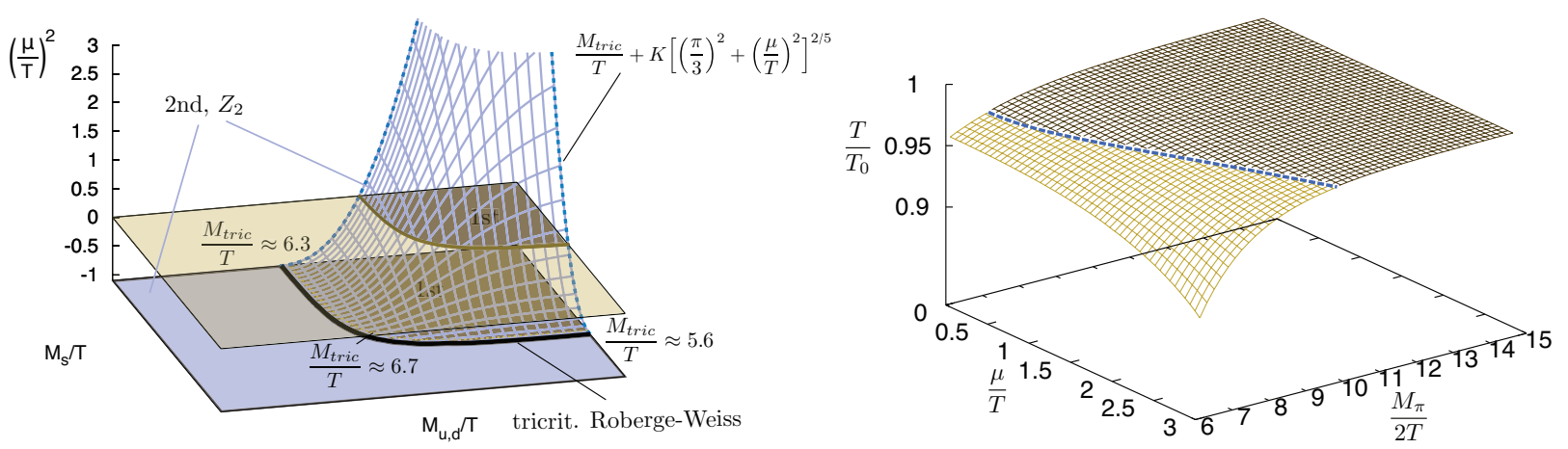

Figure 8: Left:The deconfinement critical surface of QCD with heavy quarks. Right: Phase structure of the theory in $\left(\frac{\mu}{T}, \frac{M_{\pi}}{2 T}, \frac{T}{T_{0}}\right)$-space, for $N_{f}=2$ and $N_{\tau}=6$. Above the surface the theory is deconfined. The critical line (dashed) separates the cross-over (light) and the first-order surface (dark).

Using the leading order expression for $h$, Eq. (3.4), this gives the $\bar{h}=0$-critical curve $\tilde{h}_{c}(\mu / T)$ which is also plotted in Fig. 7. Already for $\mu / T \gtrsim 1.5$ the data are accurately described by the asymptotic density limit. Similarly, the linearity of the phase boundary $\lambda(\tilde{h})$ and the near $\mu$ independence of the endpoint $\lambda_{c}$, Eqs. $(3.10,3.14)$, imply that $\tilde{h}_{c} \sim \cosh ^{-1}(\mu / T)$ [16]. We thus have obtained a numerical as well as simple parametric description of the $N_{f}=1$ deconfinement critical line for all real chemical potentials!

\section{Conclusions}

We have presented a proposal for a two-stage treatment of QCD at finite temperature and bayon density. The first step consists of an analytic derivation of a $3 \mathrm{~d}$ effective action by means of a combined strong coupling and hopping parameter expansion. The effective theory depends on Polyakov loops only, its couplings are power series of the lattice gauge coupling and hopping parameter and can be improved order by order. The second step consists of a non-perturbative Monte Carlo simulation of the effective theory. For the $S U(2)$ and $S U(3)$ pure gauge theory, the order of the deconfinement transition is correctly predicted and a continuum extrapolation of the critical temperature is feasible, within about $10 \%$ of the known answer. For heavy fermions the current effective theory is good to about $N_{\tau} \sim 6$, a continuum extrapolation is not yet feasible. On the other hand, the effective theory allows for a solution of the sign problem and thus provides a description of the deconfinement transition for all chemical potentials. This provides some hope for an eventual treatment of physical QCD, but it remains a challenging task to extend this approach to light quarks.

\section{Acknowledgements}

We thank the organizers for the invitation to a stimulating workshop and acknowledge support by the German BMBF grant 06MS9150 and by the Helmholtz International Center for FAIR within the LOEWE program of the State of Hesse. 


\section{References}

[1] O. Philipsen, arXiv:1009.4089 [hep-lat].

[2] P. H. Ginsparg, Nucl. Phys. B 170 (1980) 388; T. Appelquist and R. D. Pisarski, Phys. Rev. D 23 (1981) 2305.

[3] W. Buchmüller and O. Philipsen, Nucl. Phys. B 443 (1995) 47 [arXiv:hep-ph/9411334]; K. Kajantie, M. Laine, K. Rummukainen and M. E. Shaposhnikov, Nucl. Phys. B 466 (1996) 189 [hep-lat/9510020].

[4] F. Csikor, Z. Fodor and J. Heitger, Phys. Rev. Lett. 82 (1999) 21 [hep-ph/9809291].

[5] K. Kajantie, M. Laine, K. Rummukainen and M. E. Shaposhnikov, Nucl. Phys. B 503 (1997) 357 [arXiv:hep-ph/9704416].

[6] A. Vuorinen and L. G. Yaffe, Phys. Rev. D 74 (2006) 025011 [arXiv:hep-ph/0604100]; R. D. Pisarski, Phys. Rev. D 74 (2006) 121703 [arXiv:hep-ph/0608242].

[7] Ph. de Forcrand, A. Kurkela and A. Vuorinen, Phys. Rev. D 77 (2008) 125014 [arXiv:0801.1566 [hep-ph]]; C. Wozar, T. Kaestner, A. Wipf and T. Heinzl, Phys. Rev. D 76 (2007) 085004 [arXiv:0704.2570 [hep-lat]].

[8] M. Gross and J. F. Wheater, Nucl. Phys. B 240 (1984) 253.

[9] J. Langelage, S. Lottini and O. Philipsen, JHEP 1102 (2011) 057 [Erratum-ibid. 1107 (2011) 014] [arXiv:1010.0951 [hep-lat]].

[10] I. Montvay and G. Münster, "Quantum fields on a lattice," Cambridge, UK: Univ. Pr. (1994).

[11] J. M. Drouffe and J. B. Zuber, Phys. Rept. 102, 1 (1983).

[12] J. Polonyi and K. Szlachanyi, Phys. Lett. B 110 (1982) 395.

[13] J. Fingberg, U. M. Heller and F. Karsch, Nucl. Phys. B 392 (1993) 493.

[14] J. Kogut, M. Stone and H. W. Wyld, Phys. Rev. Lett. 50 (1983) 393.

[15] S. Necco and R. Sommer, Nucl. Phys. B 622 (2002) 328.

[16] M. Fromm, J. Langelage, S. Lottini and O. Philipsen, arXiv:1111.4953 [hep-lat].

[17] J. Langelage, O. Philipsen, JHEP 1001 (2010) 089. [arXiv:0911.2577 [hep-lat]].

[18] J. Langelage, O. Philipsen, JHEP 1004 (2010) 055. [arXiv:1002.1507 [hep-lat]].

[19] P. Hasenfratz, F. Karsch, Phys. Lett. B125 (1983) 308.

[20] F. Green and F. Karsch, Nucl. Phys. B 238 (1984) 297.

[21] H. Saito et al. [WHOT-QCD Collaboration]. arXiv:1106.0974 [hep-lat].

[22] C. Alexandrou, A. Boriçi, A. Feo, Ph. de Forcrand, A. Galli, F. Jegerlehner, T. Takaishi, Phys. Rev. D60, 034504 (1999). [hep-lat/9811028]. 